R.R. AGISHEV
A. COMERON $^{3}$
B. GROSS
F. MOSHARY
${ }^{1}$
S. AHMED

A. GILERSON
V.A. VLASOV

R.R. AGISHEV ${ }^{1,2, \infty}$

A. COMERON

F. MOSHARY ${ }^{1}$

S. AHMED ${ }^{1}$

V.A. VLASOV ${ }^{2}$

\section{Application of the method of decomposition of lidar signal-to-noise ratio to the assessment of laser instruments for gaseous pollution detection}

\author{
${ }^{1}$ City College of the City University of New York, 140 St. \& Convent Ave., New York, NY 10031, USA \\ ${ }^{2}$ Kazan State Technical University, 10, K. Marx St., Kazan, Tatarstan 420111, Russia \\ ${ }^{3}$ Polytechnic University of Catalonia, 1-3, Jordi Girona, Barcelona 08034, Spain
}

\begin{abstract}
Received: 31 December 2003/Revised version: 22 March 2004 Published online: 3 June 2004 • C Springer-Verlag 2004

ABSTRACT A general methodology for rating both performance and potential of lidar systems used for detection of atmospheric trace constituents including pollutants and gas leaks is developed. By individually examining and decomposing the contribution of both lidar system parameters and atmospheric operating conditions on signal-to-noise-ratio, a generalized figure of merit, $V$, for lidar quality is introduced and evolved. Computer simulations based on $V$ and atmospheric parameters are carried out to determine achievable lidar performance. A simple design procedure is outlined for determination of lidar instrumentation parameters to ensure the best monitoring efficiency for a given set of initial parameters/requirements, including operation range, minimum detectable gas concentration, and so on.
\end{abstract}

PACS 42.68.Wt; 42.79.Qx; 92.60.Sz; 42.62.Fi

\section{$1 \quad$ Introduction}

Lidar designs vary widely dependent on measurement application, available components and financial constraints, and design capability [1-14]. For example, Zuev et al. [4] presented data on over a hundred differential absorption lidars (DIALs) from scientific groups all over the world. On the basis of the data assembled by that paper [4], it is possible to get a general view of operational ranges and measurement capabilities achieved for a wide variety of lidar transmitting and receiving subsystems parameters. There is, however, no straightforward way for evaluating and selecting amongst these many systems the optimum one for a particular lidar application. To carry out a quantitative analysis of efficiency and potential capabilities of a lidar system, in each case a significant number of instrumental and environmental factors must be taken into account. While it is obvious that the use of a more powerful laser transmitter, a larger-aperture receiving telescope, and/or more sensitive photodetectors will allow achievement of a greater operation range, financial considerations often limit such design considerations.

The traditional approach to the analysis of lidar capabilities, discussed, for example, in [1-3, 5, 6, 8, 10-12] as well

Fax:+1-212/650-5491, E-mail: ravil_agishev@mail.ru as in many other works, is based largely on examination of the signal-to-noise ratio (SNR) at the photodetector output. This frequently used criterion for lidar instrument efficiency is a comprehensive one, since it incorporates system parameters, the medium through which signals propagate, the external background radiation, the range factor, etc. The inclusive nature of the SNR criterion makes it convenient $[6,12]$.

However, the all-inclusive nature of the SNR criterion is also its weak point $[8,13]$, because it is difficult sometimes to separate the impact of the different components mentioned above, which is desirable to a system developer or user. For example, an increase or reduction of SNR can be caused not only by a change in a constituent component but also by changes of optical 'weather' conditions for signal propagation [5, 6,9-11], by changes of background conditions [8], or by yet other factors. If the influence of the different factors cannot be evaluated separately, this makes it difficult to evaluate either subsystems or the overall system measurement capabilities [13]. It appears that a generalized methodology for lidar performance and parameter evaluation is lacking.

For example, for estimates of the required pulse energies, system developers have to use rather complex analytical expressions and empirical formulas, which are often applicable only for a very narrow range of parameters for a specific experimental setup [15]. In view of the large variety and scale of available lidar components and instruments [4,9-11], it is difficult to conclusively choose a system design which is optimized to meet the required operation range, the dynamic range of optical parameters or gas concentrations to be measured, etc.

As a consequence, valid comparisons of different lidar systems can only be made for very similar applications, including ranges and atmospheric conditions [8]. To overcome these limitations, it is necessary to evolve criteria in more universal and dimensionless form to ensure their wider applicability to the evaluation of different lidar systems and applications.

In this regard, a proper comparison of efficiency and potential of various lidar instruments can only be realized if the conditions of the experiments to be conducted, including the optical 'weather', the external background radiation, and the range factor, are similar [13]. Therefore, when formulating criteria for rating both efficiency and potential capability of given lidar instruments, it is necessary to represent them in 
universal and dimensionless form to ensure the applicability of such criteria under different conditions of operation and application.

The purpose of the present work is to evolve and generalize criteria that can be widely used to evaluate a broad range of lidar system capabilities for a variety of atmospheric trace constituent measurement applications and, based on these criteria, develop a methodology for selection of appropriate lidar system parameters that best respond to the specific application. To be able to achieve a methodology that can address the range of requirements, including the ability to handle the necessary dynamic range of concentrations at the desired distances, it is necessary to evolve a format for expressing lidar system parameters in a generalized manner that can be applied to differing systems.

2

2.1

\section{Signal-to-noise ratio (SNR) at the output of a generalized subsystem of direct photodetection} General considerations

The SNR at the output of the generalized subsystem of direct photodetection with internal gain and external and internal noise is frequently represented as follows $[5,6,10]$ :

$\varrho_{\text {out }}=\frac{P_{\mathrm{s}}}{\sqrt{\frac{2 h c \Delta f F}{\eta \lambda}\left(P_{\mathrm{s}}+P_{\mathrm{b}}\right)+\mathrm{NEP}^{2} \Delta f}}$,

where $P_{\mathrm{s}}$ is the echo-signal power reaching the photodetector, $P_{\mathrm{b}}$ is the power of the background radiation, $\eta$ is the photodetector quantum efficiency, $F$ is the excess-noise factor of the photodetector, $\Delta f$ is the receiver electrical bandwidth, and NEP is the noise-equivalent power not depending on signal and background radiation,

$\mathrm{NEP}=\frac{h c}{\eta e \lambda} \sqrt{2 e F I_{\mathrm{d}}+\frac{4 k T}{M^{2} R_{\mathrm{e}}}}$.

Here $I_{\mathrm{d}}$ is the photodetector dark current for $M=1, M$ is the actual photodetector multiplication gain, and $R_{\mathrm{e}}$ is the equivalent load of the photodetector, including the output impedance of the photodetector, the load resistor, and the input impedance of the transimpedance amplifier. For photodetectors with internal gain $M \gg 1$ the noise-equivalent power can be approximated by NEP $\cong(h c / \eta \lambda) \sqrt{2 F I_{\mathrm{d}} / e}$.

The background radiation power can be written as $[5,6]$

$P_{\mathrm{b}}=B_{\lambda} A_{\mathrm{r}} \Omega \Delta \lambda \xi_{0}$

where $B_{\lambda}$ is the spectral radiance of the zone of the sky falling in the receiver field of view, $A_{\mathrm{r}}$ the effective area of the optical receiving system, $\Omega$ the field-of-view solid angle, $\Delta \lambda$ the spectral width of the optical band-pass filter in the receiving system, and $\xi_{0}$ the optical receiver transmittivity.

\section{2}

\section{Signal threshold power at the photodetector input}

For a given signal-to-noise ratio $\varrho_{\text {out }}$ at the photodetector output, from (1), we can obtain the relative echosignal power $\psi_{\mathrm{q}}=P_{\mathrm{s}} / P_{\mathrm{q}}$, normalized to the quantum noise power $P_{\mathrm{q}}=2 h c \Delta f F / \eta \lambda$ that characterizes the quantum limit of the detector sensitivity or the threshold power at the input determined by the shot (quantum) noise:

$$
\begin{aligned}
\varrho_{\mathrm{out}}^{2} & =\frac{P_{\mathrm{s}}^{2}}{\frac{2 h c \Delta f F}{\eta \lambda}\left(P_{\mathrm{s}}+P_{\mathrm{b}}\right)+\mathrm{NEP}^{2} \Delta f} \\
& =\frac{P_{\mathrm{s}}^{2}}{P_{\mathrm{q}}\left(P_{\mathrm{s}}+P_{\mathrm{b}}\right)+\mathrm{NEP}^{2} \Delta f} \\
& =\frac{P_{\mathrm{s}}^{2} / P_{\mathrm{q}}^{2}}{\frac{P_{\mathrm{s}}+P_{\mathrm{b}}}{P_{\mathrm{q}}}+\frac{\mathrm{NEP}^{2} \Delta f}{P_{\mathrm{q}}^{2}}}=\frac{\left(P_{\mathrm{s}} / P_{\mathrm{q}}\right)^{2}}{\frac{P_{\mathrm{s}}+P_{\mathrm{b}}}{P_{\mathrm{q}}}+\frac{P_{\mathrm{n}}}{P_{\mathrm{q}}}} .
\end{aligned}
$$

Then

$\psi_{\mathrm{q}}^{2}-\varrho_{\text {out }}^{2} \psi_{\mathrm{q}}-\varrho_{\text {out }}^{2}\left(N_{\mathrm{b}}+N_{\mathrm{n}}\right)=0$,

where $N_{\mathrm{b}}=P_{\mathrm{b}} / P_{\mathrm{q}}$ is the relative power of the background clutter normalized to the quantum noise power, $N_{\mathrm{n}}=P_{\mathrm{n}} / P_{\mathrm{q}}$ is the relative power of the photodetector internal noise being referred to the input, that is, normalized to the quantum noise power, and $P_{\mathrm{n}}=\mathrm{NEP}^{2} \Delta f / P_{\mathrm{q}}$ is the power of the internal noise of the photodetector being referred to the photodetector input. Then the normalized limit of the sensitivity is

$\psi_{\mathrm{q}}^{\min }=\frac{1}{2} \varrho_{\text {out }}^{2}\left(1+\sqrt{1+4 \frac{N_{\mathrm{b}}+N_{\mathrm{n}}}{\varrho_{\text {out }}^{2}}}\right)$.

From here the absolute threshold signal power $P_{\mathrm{t}}$ (or the power of the minimal detectable signal $P_{\mathrm{s}}^{\min }$ ) is given by

$P_{\mathrm{s}}^{\min } \equiv P_{\mathrm{t}}=\frac{1}{2} \varrho_{\text {out }}^{2} P_{\mathrm{q}}\left(1+\sqrt{1+4 \frac{N_{\mathrm{b}}+N_{\mathrm{n}}}{\varrho_{\mathrm{out}}^{2}}}\right)$.

Equation (3) determines the threshold signal power at the input of the direct photodetection subsystem. From here, one can easily obtain particular cases corresponding to modes limited by background, by dark current (which determines the value of NEP), and by quantum noise. For example, in absence of the background clutter,

$P_{\mathrm{t}}^{B=0}=\frac{1}{2} \varrho_{\text {out }}^{2} P_{\mathrm{q}}\left(1+\sqrt{1+\frac{4}{\varrho_{\text {out }}^{2}} \frac{P_{\mathrm{n}}}{P_{\mathrm{q}}}}\right)$.

In other words, $P_{\mathrm{t}}^{B=0}$ is the noise power of both the photodetector's own noise and shot noise caused by the signal referred to the optical input.

In case of heterodyne detection the threshold power is determined according to papers $[5,6,9,10]$ as $P_{\mathrm{t}}=\varrho^{2} P_{\mathrm{q}} / 2$.

\subsection{Presentation of the background radiation as multiplicative clutter and estimating its influence}

Frequently for aerosol lidars with moderate pulse energies, and in all cases of Raman lidar applications, the most important factor that limits the detection of weak signals in daytime is background sky radiation. Its influence on the resulting signal-to-noise ratio is now analyzed. 
Using (2), it is easy to see that

(a) if $N_{\mathrm{b}}+N_{\mathrm{n}} \ll 1$, then $\psi_{\mathrm{q}}^{\mathrm{min}}=\varrho_{\text {out }}^{2}$,

(b) if $N_{\mathrm{b}}+N_{\mathrm{n}} \approx 1$, then $\psi_{\mathrm{q}}^{\mathrm{min}}=(1+\sqrt{5}) \varrho_{\text {out }}^{2}$, and

(c) if $N_{\mathrm{b}}+N_{\mathrm{n}}>1$ (in practice), then the ratio $\psi_{\mathrm{g}}^{\min }$ of the minimum detected signal power to the power of quantum noise is

$\psi_{\mathrm{q}}^{\min }=\frac{1}{2} \varrho_{\text {out }} \sqrt{1+4 N_{\mathrm{n}}} \sqrt{1+\frac{N_{\mathrm{b}}}{N_{\mathrm{n}}}}=\frac{1}{2} \varrho_{\text {out }} \sqrt{1+4 N_{\mathrm{n}}} U_{\mathrm{b}}$

when aiming to consider the background radiation as a multiplicative clutter. Here the background factor

$U_{\mathrm{b}}=\sqrt{1+\frac{P_{\mathrm{b}}}{P_{\mathrm{n}}}}$

takes into account the influence of the background clutter on the photodetector threshold power $P_{\mathrm{s}}^{\min }$. By introducing to (5) powers of both internal and quantum noise $P_{\mathrm{n}}$ and $P_{\mathrm{q}}$, which are referred to the photodetector input, the threshold power normalized to its shot noise is

$\psi_{\mathrm{q}}^{\min }=\frac{1}{2} \varrho_{\text {out }} \sqrt{1+4 \frac{P_{\mathrm{n}}}{P_{\mathrm{q}}}} U_{\mathrm{b}}$

Therefore, the final expression for the power of the minimum detected signal, or the threshold power of the photodetector, is given by

$P_{\mathrm{t}}=\frac{1}{2} \varrho_{\text {out }} P_{\mathrm{q}} \sqrt{1+4 \frac{P_{\mathrm{n}}}{P_{\mathrm{q}}}} U_{\mathrm{b}}$.

An illustration of the degradation of the threshold sensitivity of the generalized photodetector is shown in Fig. 1. As can be seen from Fig. 1, in the case of $P_{\mathrm{b}} / P_{\mathrm{n}} \leq 1$ the influence of the background clutter is very weak. But, the threshold power considerably worsens with the increase of the ratio $P_{\mathrm{b}} / P_{\mathrm{n}}$.

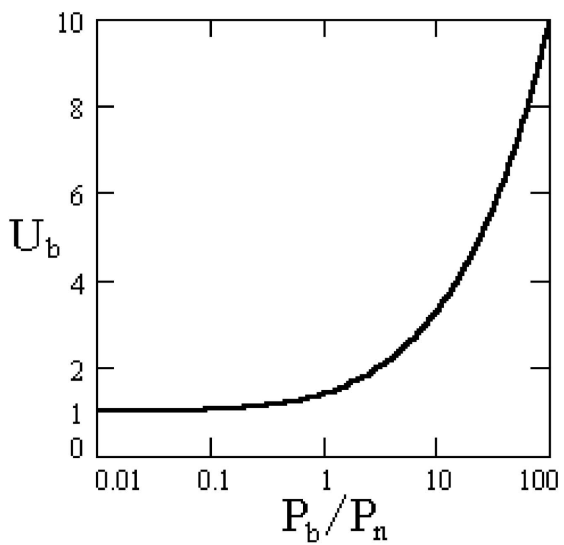

FIGURE 1 Influence of the photodetector's input SNR on the output SNR for the ratio $P_{\mathrm{b}} / P_{\mathrm{n}}=$ var

3

\section{Decomposition of the signal-to-noise ratio for} lidars

The estimates of the signal-to-noise situation developed above at the input of the generalized optical receiver pave the way for the development of a methodology for assessing the potential of lidar systems for the remote detection of atmospheric trace constituents.

\subsection{Spatial efficiency of both atmospheric and topographic backscatter lidars}

To achieve a united approach to the analysis and evaluation of lidar systems that may be operating on different schemes, for instance atmospheric backscatter lidar and topographic backscatter lidar (from remote topographic targets), we write the lidar equation in the following form:

$P_{\mathrm{S}}(\lambda, R)=K(R) P_{0}(\lambda) \xi(\lambda) T^{2}(\lambda, R)$,

where $P_{\mathrm{s}}$ is the instantaneous value of the echo-signal power received at wavelength $\lambda$ from the current range $R, K$ is the spatial efficiency of the instrument, $P_{0}$ is the power of the laser-emitted pulse, $\xi$ is the lidar optical efficiency (transmittivity), and $T$ is the atmospheric transmittance coefficient (transparency).

It is easy to show that a common lidar equation written in a single-scattering approximation,

$P_{\mathrm{s}}(\lambda, R)=\frac{1}{2} P_{0} c \tau_{\mathrm{p}} \beta_{\pi}(\lambda, R) A_{\mathrm{r}} T^{2}(\lambda, R) \xi(\lambda) R^{-2}$,

where $c$ is the velocity of light, $\tau_{\mathrm{p}}$ is the laser-pulse duration and $\beta_{\pi}$ is the volumetric backscatter coefficient, can be easily transformed to the form of (9). In this case, a factor of efficiency of the backscatter lidar $K(R)$ is defined as

$K \equiv K_{\mathrm{BS}}=\frac{1}{2} c \tau_{\mathrm{p}} \beta_{\pi} A_{\mathrm{r}} R^{-2}$,

where $\frac{1}{2} c \tau_{\mathrm{p}} \beta_{\pi}$ is the share of the backscattered optical power and $A_{\mathrm{r}} R^{-2}=\Omega_{\mathrm{r}}$ is the solid angle subtended by the receiving aperture for backscatter from range $R$.

The spatial efficiency of the lidar instrument with a remote reflector (LRR) $K \equiv K_{\text {ret }}$ can be better expressed as

$K \equiv K_{\text {ret }}=f_{\mathrm{r}}^{m} \varepsilon^{n} \gamma^{p}$.

Equation (11) is essentially a simplification of recommendations proposed by Hinkley [5]. In (11) $f_{\mathrm{r}}$ is the parameter characterizing the law of the radial distribution of the laserbeam power (usually $f_{\mathrm{r}} \sim 1$ ), $\varepsilon$ is the normalized area of the reflector $\left(\varepsilon=A_{\text {ret }} / \theta_{0}^{2} R^{2}\right.$, where $A_{\text {ret }}$ is the area of the reflector), $\theta_{0}=\lambda / A_{\mathrm{t}}^{1 / 2}$ is the transmitter angle of divergence, and $\gamma=A_{\mathrm{r}} / A_{\mathrm{t}}$, where $A_{\mathrm{t}}$ is the area of the output pupil of the transmitting optics. A numbers combination $\boldsymbol{m n \boldsymbol { n }}$ gets values $000,110,111$, and 221 , where the combination 000 corresponds to a case $\{\varepsilon>1, \varepsilon \gamma>1\}$ when the laser beam with the diffraction divergence is within the areas of both reflector and receiving optics. The combination 110 is realized at $\{\varepsilon>1, \varepsilon \gamma<1\}$ when the laser beam is within the receiving optics, but exceeds the size of the reflector. The combination 111 is for a case $\{\varepsilon<1, \varepsilon \gamma>1\}$ when the beam is within the reflector, but exceeds the size of the receiving optics. Lastly, the combination 221 is realized at $\{\varepsilon<1, \varepsilon \gamma<1\}$ 
when the laser beam exceeds both reflector and receiving optics.

In many cases, when the laser beam falls within the remote reflector, but exceeds the size of the input pupil of the receiving subsystem (i.e. the combination of parameters is $\boldsymbol{m n} \boldsymbol{p}=110$ ), the total spatial efficiency factor is

$K_{\text {ret }}=f_{\mathrm{r}} A_{\mathrm{r}} A_{\text {ret }} R^{-2} / A_{\mathrm{t}} \theta_{0}^{2}=f_{\mathrm{r}} \Omega_{\mathrm{r}} A_{\text {ret }} / A_{\mathrm{t}} \theta_{0}^{2}$.

If the lidar uses echo signals reflected from a topographical target, then according to [5] one can write

$K \equiv K_{\text {top }}=\varrho_{\mathrm{a}} A_{\mathrm{r}} / \pi R^{2}=\left(\varrho_{\mathrm{a}} / \pi\right) \Omega_{\mathrm{r}}$,

where $\varrho_{\mathrm{a}}$ is the albedo of the topographical target.

Thereby, for all lidar schemes considered, we can write the received signal in the form of (9).

\section{2}

\section{Universal parameters for system assessment}

To estimate the potential of the different lidar systems and to compare the efficiency of different technical approaches, it is necessary to select a suitable generalized lidar parameter as the criterion for comparison and evaluation. On the one hand, such a parameter should be able to characterize specific lidar systems, and on the other hand it needs to be useful for practical comparisons of different lidar systems, under the assumption of appropriate reference media of propagation and reference backscatter. The standard molecular atmosphere will be assumed here as the appropriate universal reference test medium. For these purposes, the volumetric extinction coefficient of a standard molecular atmosphere at normal conditions is $\alpha_{0}=0.011 \mathrm{~km}^{-1}(\lambda=0.55 \mu \mathrm{m})$ and the scattering indicatrix $i_{\varphi 0}$ of the molecular (Rayleigh) atmosphere for an angle $\varphi$ is equal to $i_{\varphi 0}=3\left(1+\cos ^{2} \varphi\right) / 16 \pi[5,6]$. Note that for the scattering angle $\varphi=\pi$ the value of the scattering indicatrix $i_{0} \equiv i_{\varphi 0}(\gamma=\pi)=3 / 8 \pi$.

Finally, normalizing the operating range of the lidar systems, $R$, on the basis of a selected range $R_{0}$ allows the use of a dimensionless range factor $r=R / R_{0}$ for convenient comparison. The absolute value of $R_{0}$ can be selected depending on specific requirements of the lidar application. For example, when using bistatic lidar, in most cases it is convenient to pick $R_{0}$ equal to the minimum sounded range $R_{\min }$.

We can then normalize the lidar signal $P_{\mathrm{s}}\left(\lambda, \beta_{\pi}, \alpha, R\right)$ on the basis of the echo signal $P_{\mathrm{s} 0}\left(\lambda, i_{0}, \alpha_{0}, R_{0}\right)$ received from the range $R_{0}$ at conditions of the standard molecular atmosphere characterized by the optical parameters $i_{0}$ and $\alpha_{0}$. We will then obtain

$$
\frac{P_{\mathrm{S}}\left(\lambda, \beta_{\pi}, \alpha, R\right)}{P_{\mathrm{s} 0}\left(\lambda, i_{0}, \alpha_{0}, R_{0}\right)}=\frac{K(\lambda, R) P_{0}(\lambda) T^{2}(\lambda, \alpha, R)}{K\left(\lambda, R_{0}\right) P_{0}(\lambda) T_{0}^{2}\left(\lambda, \alpha_{0}, R_{0}\right)} .
$$

Here $T_{0} \equiv T_{0}\left(\lambda, \alpha_{0}, R_{0}\right)$ is the transmittivity of the molecular atmosphere.

Let us turn to (9) and (10), and rewrite the ratio $P_{\mathrm{s}}(R) / P_{\mathrm{s} 0}$ by taking into account the parameters introduced: $R_{0}^{2}, \beta_{0}$, and
$T_{0}^{2}$ for specific parameters of a lidar system. For the backscattering lidar (BSL) we have

$$
\begin{aligned}
\left(\frac{P_{\mathrm{s}}}{P_{\mathrm{s} 0}}\right)_{\mathrm{BS}} & =\frac{\left[\frac{1}{2} P_{0} c \tau_{\mathrm{p}} \beta_{\pi}(R) A_{\mathrm{r}} T^{2}(\lambda, R) \xi R^{-2}\right]}{\left[\frac{1}{2} c P_{0} \tau_{\mathrm{p}} A_{\mathrm{r}} \xi i_{0} \alpha_{0} T_{0}^{2} R_{0}^{-2}\right]} \\
& =\left[\frac{\beta_{\pi}(R)}{i_{0} \alpha_{0}}\right]\left[\frac{T(\alpha, R)}{T_{0}\left(R_{0}\right)}\right]^{2}\left(\frac{R}{R_{0}}\right)^{-2} .
\end{aligned}
$$

For a lidar with a reflector (LWR), using (9) and (12) we obtain

$$
\begin{aligned}
\left(\frac{P_{\mathrm{s}}}{P_{\mathrm{s} 0}}\right)_{\text {ret }} & =\frac{\left[\frac{f_{\mathrm{r}} A_{\mathrm{r}} A_{\mathrm{ret}} R^{-2}}{A_{\mathrm{t}} \theta_{0}^{2}}\right] P_{0}(\lambda) \xi(\lambda) T^{2}(\lambda, R)}{\left\{\left[f_{\mathrm{r}} A_{\mathrm{r}} A_{\mathrm{ret}} R_{0}^{-2} / A_{\mathrm{t}} \theta_{0}^{2}\right] P_{0}(\lambda) \xi(\lambda) T_{0}^{2}\right\}} \\
& =\left[\frac{T(\alpha, R)}{T_{0}\left(R_{0}\right)}\right]^{2}\left(\frac{R}{R_{0}}\right)^{-2} .
\end{aligned}
$$

3.3

\section{Estimates of the signal-to-noise ratio at the photodetector input based on generalized lidar parameters}

To estimate the limits of the lidar systems for remote sensing applications, we consider the signal-to-noise ratio (SNR) at the input of the lidar receiver, when the SNR is presented as a ratio of the lidar echo-signal power to the threshold power defined by the background clutter and the photodetector internal noise is referred to the input. Taking into account (9) and (8) one can obtain

$\psi=\frac{P_{\mathrm{s}}(R)}{P_{\mathrm{t}}}=\frac{K(\lambda, R) P_{0}(\lambda) T^{2}(\lambda, R)}{\frac{1}{2} \varrho_{\text {out }} P_{\mathrm{q}} \sqrt{1+4 \frac{P_{\mathrm{n}}}{P_{\mathrm{q}}}} U_{\mathrm{b}}}$.

Here we should note once again that when the ratio $P_{\mathrm{b}} / P_{\mathrm{n}} \leq 1$, the effect of both the background radiation and the $U_{\mathrm{b}}$ factor on the value of $\psi$ is negligible (i.e. $U_{\mathrm{b}}=1$ ), as was explained in Sects. 2.2 and 2.3. We also take into account that the efficiency of spatial filtration of the background clutter has been studied in detail in our earlier paper [13].

We now return to (9) and (10) and rewrite the input SNR by taking into account the $R_{0}^{2}, \beta_{0}$, and $T_{0}^{2}$ values introduced. For the backscattering lidar (BSL) we shall have according to (15) and (16)

$\psi_{\mathrm{BS}}=\left(\frac{1}{2} c P_{0} \tau_{\mathrm{p}} A_{\mathrm{r}} \xi i_{0} \alpha_{0} T_{0}^{2} R_{0}^{-2}\right) \frac{\left[\frac{\beta_{\pi}(R)}{i_{0} \alpha_{0}}\right]\left(\frac{T}{T_{0}}\right)^{2}\left(\frac{R}{R_{0}}\right)^{-2}}{P_{\mathrm{t}}}$.

For a lidar with a reflector (LWR), when $\beta_{0}=1$, we obtain

$\psi_{\mathrm{ret}}=\left(\frac{\xi f_{\mathrm{r}} A_{\mathrm{r}} A_{\mathrm{ret}} P_{0} T_{0}^{2} R_{0}^{-2} A_{\mathrm{t}}^{-1} \theta_{0}^{-2}}{P_{\mathrm{t}}}\right)\left(\frac{T}{T_{0}}\right)^{2}\left(\frac{R}{R_{0}}\right)^{-2}$.

We now introduce the system parameter $V$ as the ratio between the echo-signal power, received from the reference range $R_{0}$ under the assumption of a standard molecular atmosphere (i.e. $i_{0}$ and $\alpha_{0}$ ) and the threshold power when the 
background noise is negligible $\left(U_{\mathrm{b}}=1\right)$. Using (18) and (19) we obtain for BSL:

$V_{\mathrm{BS}} \equiv \frac{P_{\mathrm{s} 0}\left(i_{0}, \alpha_{0}, R_{0}\right)}{P_{\mathrm{t}}}=\frac{\frac{1}{2} P_{0} c \tau_{\mathrm{p}} i_{0} \alpha_{0} T_{0}^{2} A_{\mathrm{r}} \xi R_{0}^{-2}}{\left[\frac{1}{2} \varrho_{\text {out }} P_{\mathrm{q}} \sqrt{1+4 \frac{P_{\mathrm{n}}}{P_{\mathrm{q}}}}\right]}$.

The corresponding result for LWR is

$V_{\text {ret }} \equiv \frac{P_{\mathrm{s} 0}\left(\alpha_{0}, R_{0}\right)}{P_{\mathrm{t}}}=\frac{\xi f_{\mathrm{r}} P_{0} A_{\mathrm{r}} A_{\text {ret }} T_{0}^{2} R_{0}^{-2}}{\theta_{0}^{2} A_{\mathrm{t}}\left[\frac{1}{2} \varrho_{\text {out }} P_{\mathrm{q}} \sqrt{1+4 \frac{P_{\mathrm{n}}}{P_{\mathrm{q}}}}\right]}$.

Now we will introduce one more parameter $W$, taking into account (18) and (19):

$W=b\left(\frac{T}{T_{0}}\right)^{2}$

with $b=\beta_{\pi} / i_{0} \alpha_{0}$ for BSL and $b=1$ for LWR. Then for BSL and LWR correspondingly we have

$W_{\mathrm{BS}}=\left(\beta_{\pi} / i_{0} \alpha_{0}\right)\left[\frac{T(\lambda, \alpha, R)}{T_{0}\left(\lambda, \alpha_{0}, R_{0}\right)}\right]^{2}$,

$W_{\text {ret }}=\left[\frac{T(\lambda, \alpha, R)}{T_{0}\left(\lambda, \alpha_{0}, R_{0}\right)}\right]^{2}$.

\subsection{Interpretation of the parameters $V, U_{b}, W$, and $r$}

For both types of lidar considered, we took the input signal-to-noise ratio $\psi$ as a product of dimensionless normalized multipliers

$\psi=V U_{\mathrm{b}}^{-1} W r^{-2}$.

The first multiplier represents an energy component $V$ of the total input signal-to-noise ratio for the echo signal received from the range $R_{0}$ under conditions of a standard molecular atmosphere. This is an essential distinctive feature of $V$. That is why the dimensionless parameter $V$ can be considered as a universal criterion for comparison of potential and performance of different lidar systems. As can be seen from (20) and (21), to be able to calculate the value of the parameter $V$ for either an existing lidar system or one under development, it is necessary to know only parameters of both transmitting and receiving subsystems of the lidar and also to introduce the optical parameters of a standard molecular atmosphere.

From (20) and (21) it can be estimated that the value of $V$ varies between rather wide limits: from $10^{-12}$ up to $10^{3}$ for the backscattering lidar (BSL) and from $10^{0}$ up to $10^{7}$ for the lidar with remote reflector (LWR) for wide variations of both transmitting and receiving subsystems parameters $\left(P_{0}, \tau_{\mathrm{p}}, A_{\mathrm{r}}, \xi, R_{0}, \mathrm{NEP}\right.$, and $\left.\Delta f\right)$. The shift of the $V$ parameter variability range for the LWR to larger values in comparison with the BSL is determined by a significantly larger albedo of the reflector or the topographical target as compared to the aerosol-scattering coefficient.

It is easy to see that the large values of $V$, on the one hand, mean a high performance of the lidar; on the other hand, they correspond to an increased cost for higher-quality equipment.
The second multiplier of (24) characterizes the influence of the background clutter to the lidar threshold power according to (5) and (6).

The third multiplier of (24) is a normalized atmospheric component $W$ of the input SNR. $W$ is determined by the difference of the current optical weather state from the standard molecular atmosphere. The parameter $W$ includes information about the concentration of the trace gases under investigation.

The fourth multiplier of (24) is a normalized range factor that allows comparing the current range $R$ with the initial range $R_{0}$.

Finally, it should be pointed out that to keep the role and the advantages of different lidar types, the parameterization utilized in this paper can be extended to micro-pulse lidars [16, 17], which use high pulse rate and low pulse energy. In this case, the $V$ parameter may be rescaled as $V_{\text {eq }}=V \sqrt{N}$, where $\sqrt{N}$ represents the improved signal-to-noise ratio and $N$ is the number of lidar signals accumulated. For a repetition frequency $f_{\text {mod }}$ and an obscuration period $T_{\mathrm{obs}}$, the number $N$ is equal to $N=f_{\bmod } T_{\mathrm{obs}}$. Then the equivalent system parameter is

$V_{\mathrm{eq}}=V \sqrt{f_{\mathrm{mod}} T_{\mathrm{obs}}}$.

With this equivalent parameterization, features and merits of different types of lidar instrument may be taken into account, and all lidars may be correctly compared.

\section{4}

\section{Application of generalized system parameters for estimations of potential capabilities of lidar}

To determine the limits of the capabilities of a lidar to detect the atmospheric constituents, it was necessary to set requirements under which the receiving echo signals will exceed the internal and external noise. In Sect. 2, we considered the main factors limiting the threshold sensitivity of the lidar receiving subsystem, and also analyzed the reduction of the SNR under the influence of external background clutter. In this section, we focus on the application of the SNR parameterization developed earlier to the case of a molecular atmosphere in both horizontal and vertical sounding geometries as well as a prototypical differential absorption lidar system.

\subsection{Backscattering lidar in molecular atmosphere}

The parameterization developed can be used to study the ultimate sounding range for an elastic lidar in both vertical and horizontal geometries. To understand the importance of this measurement, we fix our attention on the calibration aspects of the lidar. In particular, calibration of the lidar signal requires sufficient signal above the aerosol layer to match the signal to a molecular atmosphere. Therefore, connecting the $V$ parameter to the maximum range in which a molecular backscatter signal is detectable is of considerable interest.

4.1.1 Horizontal sounding. To begin, we consider horizontal sounding in the lower troposphere. From (24) it is easy to determine an operating range of the lidar, having accepted the input signal-to-noise ratio $\psi=1$ : 
$r_{\max }=\sqrt{\frac{V W\left(R_{\max }\right)}{U_{\mathrm{b}}}}$.

For pure molecular atmosphere conditions, when $i=i_{0}$ and $\alpha=\alpha_{0}$,

$$
\begin{aligned}
W_{0} & \equiv\left[\frac{\beta_{\pi}}{i_{0} \alpha_{0}}\right]\left[\frac{T(\lambda, \alpha, R)}{T_{0}\left(\lambda, \alpha_{0}, R_{0}\right)}\right]^{2} \\
& =\exp \left[-2 \alpha_{0}\left(R-R_{0}\right)\right] \\
& =\exp \left\{-2 \alpha_{0}\left[R_{0}(r-1)\right]\right\} .
\end{aligned}
$$

Therefore, the sounding range is given as the solution of a nonlinear algebraic equation, which may be easily solved in an iterative manner:

$R_{\max , i}=R_{0} \sqrt{\frac{V \exp \left[-2 \alpha_{0}\left(R_{\max , i-1}-R_{0}\right]\right.}{U_{\mathrm{b}}}}$.

Results of estimates of the backscattering lidar's normalized operation range at molecular atmosphere for horizontal sounding (the height $h \sim 0$ ) and negligible background noise $\left(U_{\mathrm{b}}=1\right)$ are given in Figs. 2 and 3.

The maximum operation range of lidars with different $V$ parameters and similar values of $R_{0}$ can be easily compared using Figs. 2 and 3a. When lidars have different $R_{0}$, one should use Fig. 3b, because the $V$ parameter depends on $R_{0}$ according to the definition (20).

If the power of background radiation is low $\left(U_{\mathrm{b}}=1\right)$ and atmospheric optical densities $\alpha_{0} R<1$, the maximum operation range can be represented as

$r_{\max }=\sqrt{V}$.

From here, a physical sense of the system parameter $V$ becomes obvious: its numerical value defines a square of a normalized operation range of the lidar at horizontal sounding and standard molecular atmosphere conditions (with $\left.\alpha_{0} R<1\right)$ in the absence of background clutter.

4.1.2 Vertical sounding. For vertical sounding, the heightdependent changes of the molecular scattering coefficient $\alpha_{0}$

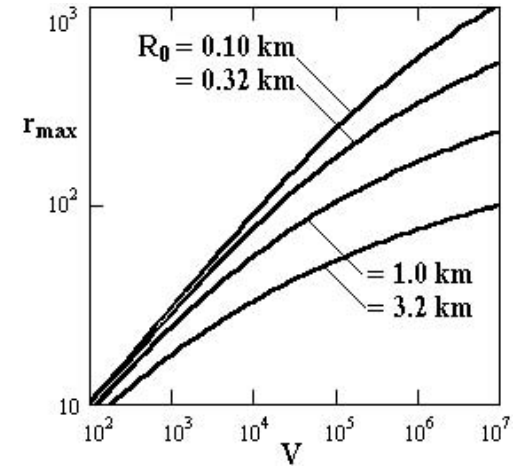

FIGURE 2 Normalized operating range $r_{\max }=R_{\max } / R_{0}$ of backscattering lidar at molecular atmosphere as a function of system parameter $V$ for different values of the initial range $R_{0}$

will significantly correct the maximum $Z_{\max }$ due to the decrease of the air-molecule concentration $N_{0}(Z)$ that can be estimated as $N_{0}(Z)=N_{0}(0) \exp \left(-Z / Z_{\mathrm{a}}\right)$ [6]. Here $Z$ is the vertical height, $Z_{\mathrm{a}} \approx 8 \mathrm{~km}$ is the atmospheric scale height, and $N_{0}(0)$ is the standard atmospheric air concentration at ground level.

Then (25) can be written as follows:

$z_{\max }=\sqrt{\frac{V}{U_{\mathrm{b}}} W_{0}\left(Z_{\max }\right)}$,

where the atmospheric transmittance term is

$$
\begin{aligned}
& W_{0}(Z)=\exp \left(-2 \int_{Z_{0}}^{Z} \alpha(z) \mathrm{d} z\right) \\
& =\exp \left[-2 \alpha_{0}(0) \int_{Z_{0}}^{Z} \exp \left(-z / Z_{\mathrm{a}}\right) \mathrm{d} z\right] \\
& =\exp \left\{-2 Z_{\mathrm{a}} \alpha_{0}(0) \exp \left(-\frac{Z_{0}}{Z_{\mathrm{a}}}\right)\left[1-\exp \left[(1-z) \frac{Z_{0}}{Z_{\mathrm{a}}}\right]\right]\right\},
\end{aligned}
$$

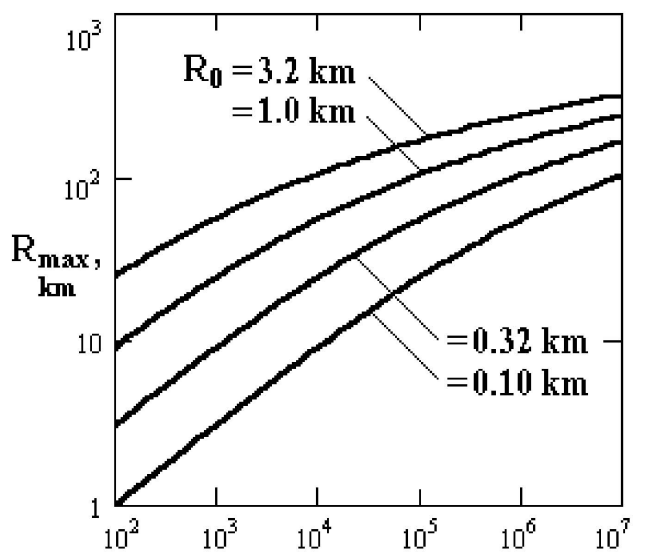

a $\mathrm{V}$

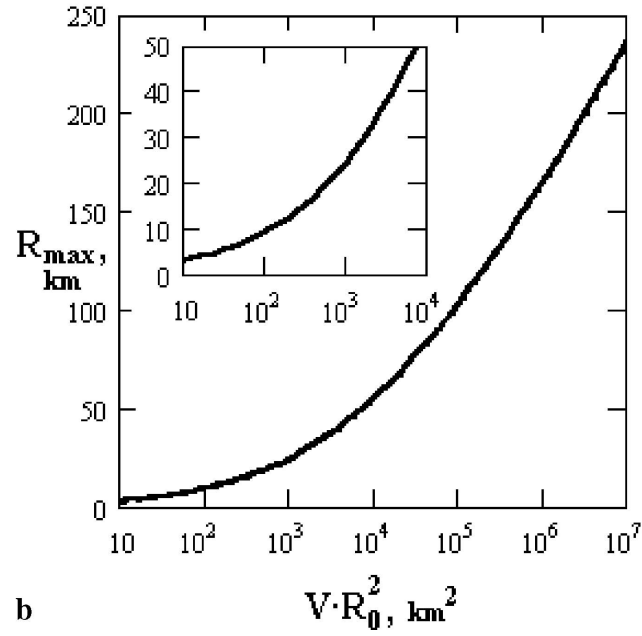

FIGURE 3 Maximum operation range $R_{\max }$ of backscattering lidar at molecular atmosphere as a function of system parameter $V$ for different values of the initial range $R_{0}(\mathbf{a})$ and as a function of parameter $V \times R_{0}^{2}(\mathbf{b})$ 
and where $Z_{0}$ is the initial height (similar to $R_{0}$ ). The illustration of the dependence $W_{0}=f(Z)$ is given in Fig. 4 .

From Fig. 4, it is clear that due to the decreasing gas concentration with height, at vertical sounding, the attenuation effects are much less than those assuming a homogeneous layer and will therefore lead to much higher ranges for the same $V$-parameter lidar.

Explicitly, the maximum relative operation range is solved as before from the transcendental equation

$z_{\max }=z_{0} \sqrt{\frac{V}{U_{\mathrm{b}}} \exp \left\{\begin{array}{c}-2 Z_{\mathrm{a}} \alpha_{0}(0) \exp \left(-\frac{Z_{0}}{Z_{\mathrm{a}}}\right) \\ \left.\times\left[1-\exp \left[\left(1-z_{\max }\right) \frac{Z_{0}}{Z_{\mathrm{a}}}\right]\right]\right\}\end{array} .\right.}$

The dependence of the relative height $z_{\max }$ vs. $V$ parameter (when supposing $U_{\mathrm{b}}=1$ ) corresponding to the case of the vertical sounding at molecular atmosphere conditions at $\lambda=0.55 \mu \mathrm{m}$ is presented in Fig. 5 .

In the calculations above, it was assumed that there were no aerosol contributions and that the detection threshold was determined by the condition that $\mathrm{SNR}=1$. However, when looking at calibrating a vertical-pointing lidar, an aerosol contribution layer within the boundary layer must be taken into account as well as the fact that a much higher signal-to-noise level must be maintained to accurately match the signal to a molecular layer. The addition of effects is accomplished by

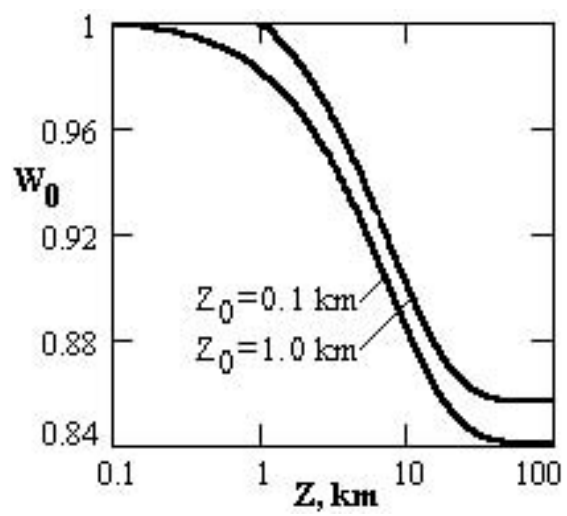

FIGURE $4 W_{0}$ parameter for the backscattering lidar at vertical sounding vs. height $Z$

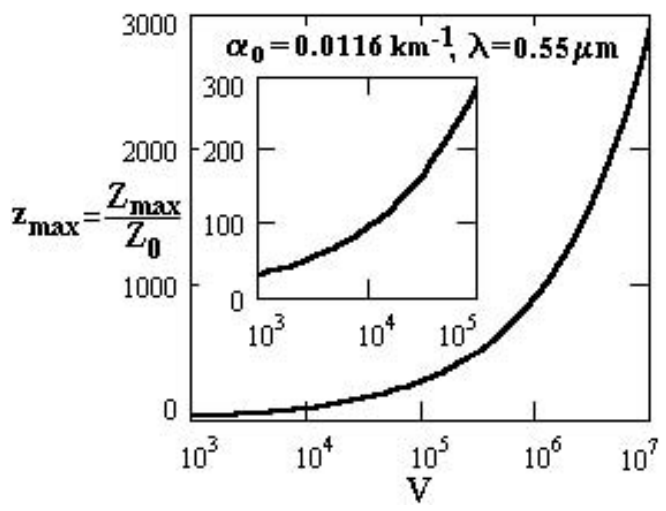

FIGURE 5 Maximum relative operation range $z_{\max }$ of the backscattering lidar at vertical sounding of the molecular atmosphere as a function of system parameter $V$ for different values of the initial height $Z_{0}$ simply redefining the $V$ parameter to account for these effects, so that $V_{\text {eff }}=\psi_{\text {des }} V / T_{\text {aer }}^{2}$, where $\psi_{\text {des }}$ represents the desired in-

put SNR and $T_{\text {aer }}$ represents the transmission of the aerosol component (treated as a constant) and can be determined from Sun photometry measurements.

\subsection{Estimation of maximum gas concentration}

To begin, we first must evaluate the upper limit of detected gas concentrations to make sure that the design is able to handle the maximum gas concentrations that can be expected. It is clear that the limit is set when the SNR given by (24) decreases to $\psi=1$ :

$\psi=V U_{\mathrm{b}} W\left(\frac{R}{R_{0}}\right)^{-2}=V b\left(\frac{T}{T_{0}}\right)^{2} r^{-2}=1$.

Let us suppose for simplicity that here $U_{\mathrm{b}}=1$. Since $R_{0} \ll$ $\alpha_{0}^{-1}$ generally, the value of $T_{0}\left(\alpha_{0}, R_{0}\right)=\exp \left(-2 \alpha_{0} R_{0}\right) \cong 1$ with a high accuracy so that we can ignore $T_{0}$. Further, we may decompose $T(\lambda, R)$ into a background atmospheric term $T_{\mathrm{a}}(\lambda, R)$ and a term for the gas under investigation $T_{\mathrm{g}}(\lambda, R)$, resulting in

$T(\lambda, R)=T_{\mathrm{g}}(\lambda, R) T_{\mathrm{a}}(\lambda, R)=\exp \left\{-\left[\tau_{\mathrm{a}}(\lambda, R)+\tau_{\mathrm{g}}(\lambda, R)\right]\right\}$,

where the optical densities are defined as

$\tau_{\mathrm{g}}(\lambda, R)=\int_{0}^{R} \alpha_{\mathrm{g}}(\lambda, r) \mathrm{d} r \quad$ and $\tau_{\mathrm{a}}(\lambda, R)=\int_{0}^{R} \alpha_{\mathrm{a}}(\lambda, r) \mathrm{d} r$.

Applying (31), the minimum detected value of the transmission coefficient along the path for a current range $R$ is

$T_{\min }(\lambda, R)=r / b^{1 / 2} V^{1 / 2}$

and, therefore, the maximum optical density of gas $\tau_{\max }$ on a path of length $R$ will be

$\tau_{\max } \equiv \max \left\{\tau_{\mathrm{g}}(R)\right\}=\ln \left(b^{1 / 2} V^{1 / 2} / r\right)-\tau_{\mathrm{a}}$.

In Fig. 6 the dependences of a maximum detected optical density of gas $\tau_{\max }$ from the operation range $R$ for lidar with a reflector $(b=1)$ are presented. As is seen from Fig. 6, for the larger values of the system parameter $V$, both the operation range for reliable detection of pollution and the upper limit of the detected optical gas density grow.

From (33) we can estimate the maximum concentration of gas under investigation when averaged over a path length $R_{\max }$ that can be measured by a chosen lidar instrument:

$N_{\max \mathrm{AV}}=\tau_{\max } / \sigma_{1} R=\left[\ln \left(b^{1 / 2} V^{1 / 2} / r\right)-\tau_{\mathrm{a}}\right] / \sigma_{1} R$,

where $\sigma_{1}$ is the cross section of absorption for the absorbing line.

As one can see from Fig. 7, though the maximum detectable concentration $N_{\max }$ decreases with range, an increased $V$ will extend the measurement range. 


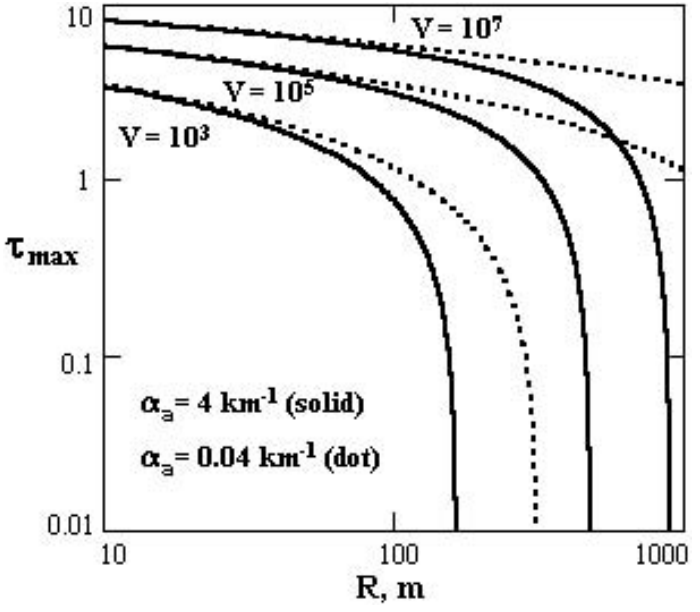

FIGURE 6 Upper limit of detectable optical density $\tau_{\max }$ vs. range $R$ when using a lidar with a remote reflector; $R_{0}=10 \mathrm{~m}$

\section{3}

\section{Estimation of minimum gas concentration}

For the differential method (DIAL, etc.), the sensitivity threshold (i.e. the lower limit of detectable concentrations) is determined by insuring that the differential signal is above (at) threshold:

$P_{\mathrm{r} 2}\left(\lambda_{2}\right)-P_{\mathrm{r} 1}\left(\lambda_{1}\right)=P_{\mathrm{t}}$ or $\left[P_{\mathrm{r} 2}\left(\lambda_{2}\right)-P_{\mathrm{r} 1}\left(\lambda_{1}\right)\right] / P_{\mathrm{t}}=1$

Assuming equal system parameters and aerosol extinction coefficients $\alpha_{\mathrm{a}}$ for $\lambda_{1}$ and $\lambda_{2}$, it is easy to obtain by using (20), (21), (22), and (23) that

$$
\begin{aligned}
& {\left[V\left(\lambda_{2}\right) W\left(\lambda_{2}\right)-V\left(\lambda_{1}\right) W\left(\lambda_{1}\right)\right]\left(\frac{R}{R_{0}}\right)^{-2}} \\
& =V b \exp \left(-2 \tau_{\mathrm{a}}\right)\left[\exp \left(-2 \tau_{\mathrm{g} 2}\right)-\exp \left(-2 \tau_{\mathrm{g} 1}\right)\right] / r^{2}=1,
\end{aligned}
$$

where the indices 1 and 2 refer to wavelengths $\lambda_{1}$ and $\lambda_{2}$, respectively. From here

$\exp \left(-2 \tau_{\mathrm{g} 2}\right)-\exp \left(-2 \tau_{\mathrm{g} 1}\right)=r^{2} / V b \exp \left(-2 \tau_{\mathrm{a}}\right)$.

By expanding the left-hand-side exponents in Taylor series and keeping for small optical densities only the two first terms of the series in the first approximation, we obtain

$$
\begin{aligned}
\tau_{\min } \equiv \Delta \tau_{\mathrm{gmin}} & =\tau_{\mathrm{g} 1}-\tau_{\mathrm{g} 2} \\
& =\Delta \alpha_{\mathrm{g}}^{\min } R=r^{2} / 2 V b \exp \left(-2 \tau_{\mathrm{a}}\right) .
\end{aligned}
$$

It follows from (35) that to detect low concentrations of gas, the system should be sensitive to very small optical densities. As one can see from Fig. 8, when the system quality parameter $V$ increases, the absolute value of the threshold sensitivity decreases, and the operation range is increased.

The minimum detectable concentration of the analyzed gas averaged over the path length $R$ can then be obtained from (35), as was done for the maximum concentration, as

$N_{\min }=\Delta \tau_{\text {gmin }} / \Delta \sigma R=r^{2} / 2 \Delta \sigma R V b \exp \left(-2 \tau_{\mathrm{a}}\right)$,

where $\Delta \sigma=\sigma_{1}-\sigma_{2}$ is the differential cross section of the gas absorption $\left[\mathrm{cm}^{2}\right]$ for wavelengths $\lambda_{1}$ and $\lambda_{2}$.

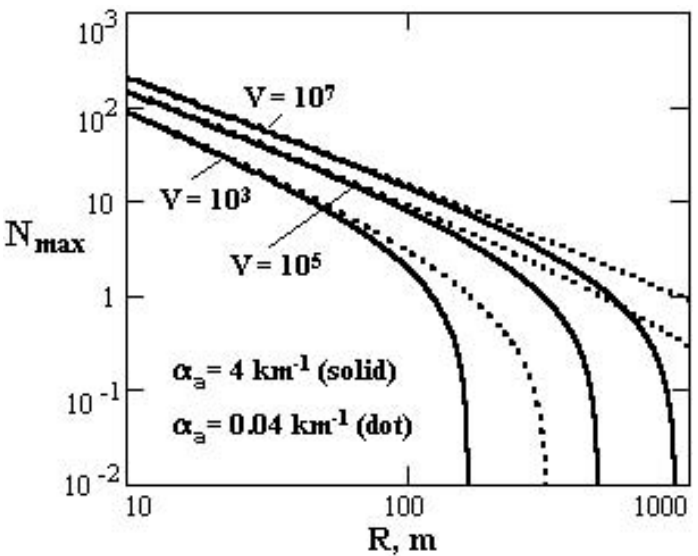

FIGURE 7 Maximum detectable gas concentration $N_{\max }$ [ppm] vs. range $R$ when using a lidar with a remote reflector; $R_{0}=10 \mathrm{~m}$

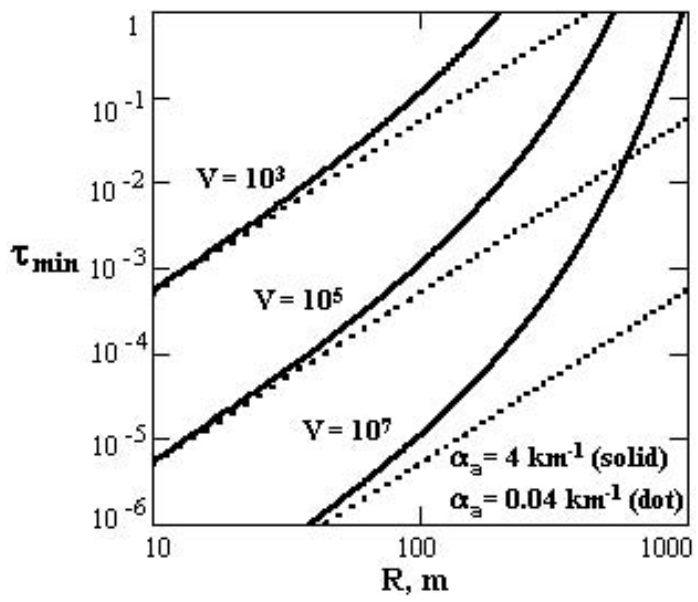

FIGURE 8 Lower limit of detectable optical density $\tau_{\min }$ vs. range $R$ when using a lidar with a remote reflector; $R_{0}=10 \mathrm{~m}$

It follows from (35) that the influence of the system parameter $V$ on the sensitivity threshold is decisive. As is seen from Fig. 9, by choosing the sounding laser with a sufficiently high radiation power and/or a good photodetector with a high detectivity, one can detect small gas concentrations on ranges of hundreds of meters. At the same time, if one only has inexpensive low-power radiators and/or photodetectors, the threshold sensitivity of the measuring system is significantly degraded (by several orders of magnitude). Thus, to estimate the average value of $N_{\min }$ it is necessary only to estimate the values of $\beta_{\pi}$ and $\alpha_{\mathrm{a}}$ once $V$ is determined.

Analyzing the behavior of the functions $\tau_{\min / \max }(V, R)$ and $N_{\min / \max }(V, R)$, one can see that the $V$ parameter is very convenient to rate the capabilities of a lidar either existing or in the design phase.

\section{5}

\section{Selection guidelines for gaseous pollution detector by using the $\tau-V$-diagram method}

In Sect. 4, we developed formulas allowing for the construction of a set of phase plots which graphically describe the lidar operation. In this section, we show how these phase plots can be used. 


\section{$5.1 \quad \tau-V$ method}

Consider the case where optimal design is based on the specification $\tau_{\mathrm{min}}^{\mathrm{req}}\left(\right.$ or $N_{\mathrm{min}}^{\mathrm{req}}$ ) for a required operation range $R_{\text {req }}$, which must also be consistent with $\tau_{\max }^{\text {req }}$. In this case, the designer must determine the lidar characteristics needed by determining the $V_{\min }$ needed. A convenient approach to do this is by combining the two families of curves $\tau_{\max }=\tau_{\max }(V)$ and $\tau_{\min }=\tau_{\min }(V)$, built for constant values of operation range ( $R=\mathrm{const}$ ), in the same graph. The procedure is illustrated in Fig. 10.

1. For a given $\tau_{\mathrm{min}}^{\mathrm{req}}$, draw a horizontal line until it intersects the $\tau_{\min }(V)$-branch curves at $R_{\text {req }}$, which is given as point A that determines an estimate of $V$.

2. From this point, draw a vertical line for value $V$ until it intersects the $\tau_{\max }(V)$ branch for the particular value of $R_{\text {req }}$ (i.e. point A1).

3. The intersection point will show the maximum achievable value of the optical density $\tau_{\max }^{\text {ach }}$.

It should be pointed out however that the procedure described above if implemented once at the specification boundaries may not work, since the resulting value of $\tau_{\max }$ may fall below the specifications needed. In such a case, the design approach may be repeated by entering the curve at a value $\tau_{\text {min,new }}<\tau_{\text {min }}$. In this case, the intersection will occur at a higher $V$ which will result in a $\tau_{\max \text {,new }}>\tau_{\max }$. This procedure can be applied until the dynamic spread is sufficient at the expense of a superior lidar design.

\section{2}

\section{$\tau-R$ method}

If the lidar system is already developed, the question of the maximum range for a given lidar to meet concentration specifications becomes relevant. In this scenario, a required threshold of detected optical density $\tau_{\min }$ and the available lidar instrument with the system parameter $V=V_{\text {avail }}$ are the input variables. A convenient approach to do this which is very similar to Sect. 5.1 is by combining the two families of curves $\tau_{\max }=\tau_{\max }(R)$ and $\tau_{\min }=\tau_{\min }(R)$, built for constant values of lidar design parameter $(V=$ const $)$, in the same graph. The details of the design procedure are illustrated in Fig. 11, where the rules are similar to the $\tau-V$ design.

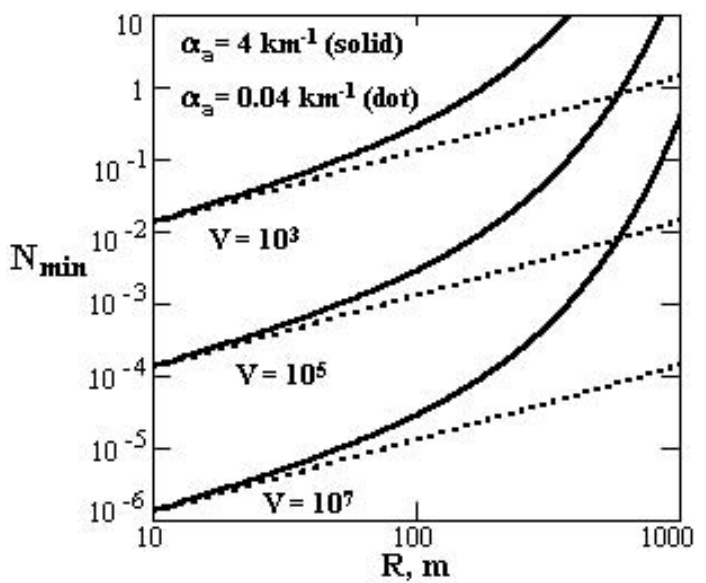

FIGURE 9 Lower limit of detectable concentration $N_{\min }$ [ppm] vs. range $R$ when using a lidar with a remote reflector; $R_{0}=10 \mathrm{~m}$

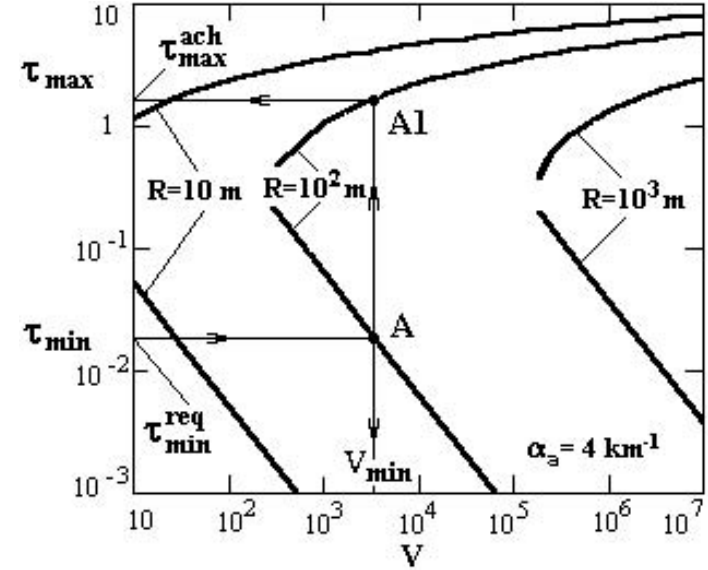

FIGURE 10 Illustration of the remote instrument selection guidelines by using the $\tau-V$ diagram

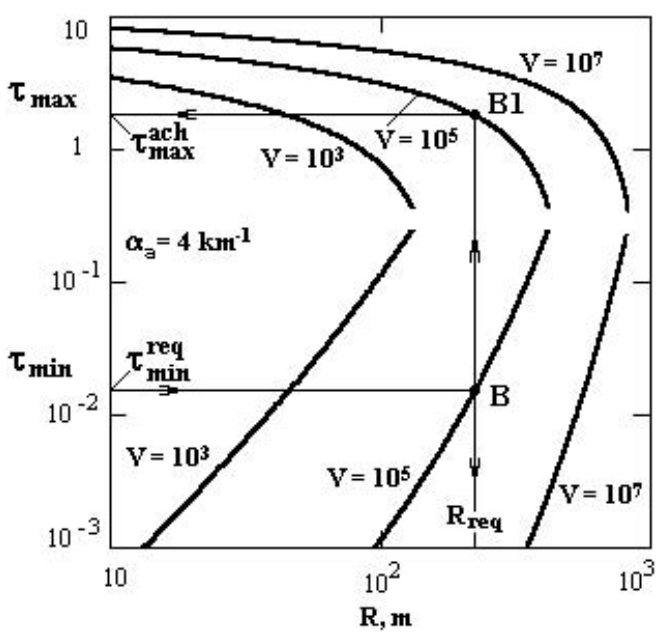

FIGURE 11 Illustration of the remote instrument selection guidelines by using the $\tau-R$ diagram

Again it should be emphasized that when first applied to the constraint specifications, $\tau_{\max }$ may not be met; in this case lowering $\tau_{\min }$ can be used to meet design specifications at the expense of a shorter gas path.

Both strategies can be used in complement to allow us to assess the detectability of gaseous species over a fixed operation range $R$ within the concentration range from $N_{\min }=$ $\tau_{\min }^{\text {req }} /\left(\Delta \sigma \cdot R_{\text {req }}\right)$ to $N_{\max }=\tau_{\max }^{\text {ach }} /\left(\sigma_{1} \cdot R_{\text {req }}\right)$ over a choice of lidar configurations.

6

\section{Conclusions}

The concept of rating both the efficiency and the potential capabilities of lidar instruments used for detection of atmospheric trace constituents, including pollution and gas leaks, was developed in this paper. Using a method of decomposition, a signal-to-noise ratio including the influence of the background clutter was presented as a product of the nondimensional energetic and atmospheric components which were normalized for standard conditions of lidar measurements. Based on the introduced generalized parameter of lidar quality $V$, the analysis and mathematical modeling of achievable concentration limits of detection for a DIAL li- 
dar system was carried out. Furthermore, an optimization method based on the introduced parameterization scheme was developed to ensure the optimum monitoring efficiency for a given set of initial parameters (operation range of gas detection, minimum detectable concentration, etc.). Further applications to elastic lidar systems in both horizontal and vertical sounding geometries were given to show the usefulness of the decomposition parameters in assessing lidar performance.

ACKNOWLEDGEMENTS The authors acknowledge partial support of this work by grants from NOAA\#NA17AE1625 and NASA\#NCC1-03009.

\section{REFERENCES}

1 U. Singh, T. Itabe, Z. Liu (Eds.): Proc. SPIE 4893, p. 1, 121 (2003)

2 K. Schaefer, O. Lado-Bordowsky, A. Comeron, R. Picard (Eds.): Proc. SPIE 4882, 400 (2003)

3 G. Kamerman (Ed.): Proc. SPIE 4723, p. 120, 156 (2002)

4 V. Zuev, M. Kataev, M. Makogon, A. Mitsel: Atmos. Ocean. Opt. 8, 1136 (1995)
5 E.D. Hinkley (Ed.): "Laser Monitoring of the Atmosphere", In: Topics in Applied Physics, Vol. 14 (Springer, Berlin 1978) p. 313

6 R.M. Measures: Laser Remote Sensing: Fundamentals and Applications (Wiley, New York 1984)

7 M. Sigrist (Ed.): Air Monitoring by Spectroscopic Techniques (Wiley, New York 1994)

8 R.R. Agishev: Protection from Background Clutter in Electro-Optical Systems of Atmosphere Monitoring (Mashinostroenie, Moscow 1994) [in Russian]

9 J. Bosenberg, D. Brassington, P. Simon (Eds.): Instrument Development for Atmospheric Research and Monitoring: Lidar Profiling, DOAS and TDLS (Springer, Berlin 1997)

10 A.D. Devir, A. Kohnle, U. Schreiber, C. Werner (Eds.): Proc. SPIE 3494, 277 (1998)

11 U. Schreiber, C. Werner, G. Kamerman, U. Singh (Eds.): Proc. SPIE 4546, p. 66, 133 (2002)

12 G.R. Osche: Optical Detection Theory for Laser Applications (Wiley, New York 2002)

13 R.R. Agishev, A. Comeron: Appl. Opt. 41, 7516 (2002)

14 R.R. Agishev, L.R. Aybatov, R.K. Sagdiev, V.A. Vlasov: Atmos. Ocean. Opt. 12, 69 (1999)

15 R. Vilar, A. Lavrov: Appl. Phys. B 71, 225 (2000)

16 J.R. Campbell, E.J. Welton, J.D. Spinhirne, Q. Ji, S.-C. Tsay, S. Piketh, M. Barenbrug, B. Holben: J. Geophys. Res. 108, 847 (2003)

17 E.J. Welton, J.R. Campbell, J.D. Spinhirne, V.S. Scott: Proc. SPIE 4153, $151(2001)$ 